The effect of irradiation and radiolytic oxidation on the porous space of

Gilsocarbon nuclear graphite measured

with mercury porosimetry and helium pycnometry

Jones, Katie Louise

http://hdl.handle.net/10026.1/15255

10.1016/j.carbon.2019.11.084

Carbon

Elsevier BV

All content in PEARL is protected by copyright law. Author manuscripts are made available in accordance with publisher policies. Please cite only the published version using the details provided on the item record or document. In the absence of an open licence (e.g. Creative Commons), permissions for further reuse of content should be sought from the publisher or author. 
"This is the author's accepted manuscript. The final published version of this work is published by Elsevier in Carbon, March 2020 available at: https://doi.org/10.1016/j.carbon.2019.11.084 This work is made available in accordance with the publisher's policies. Please refer to any applicable terms of use of the publisher."

\title{
The effect of irradiation and radiolytic oxidation on the porous space of Gilsocarbon nuclear graphite measured with mercury porosimetry and helium pycnometry
}

\author{
Katie L. Jones ${ }^{\mathrm{a}}$, G. Peter Matthews ${ }^{\mathrm{a}}$, Giuliano M. Laudone ${ }^{\mathrm{a}, *}$ \\ ${ }^{a}$ Faculty of Science and Engineering, University of Plymouth, Plymouth, UK.
}

\begin{abstract}
Gas pycnometry and mercury porosimetry are used to investigate the porous network of Gilsocarbon nuclear graphite samples that are representative of the material present in the cores of UK Advanced Gas-Cooled reactors at different stages of the reactors' operational lifetimes. Irradiation and radiolytic oxidation change the pore volume of nuclear graphite and the relative ratios of open (coolant gas accessible) and closed pore volume. Particular focus has been paid to the deformation of the Gilsocarbon graphite observed during mercury intrusion at high pressure, which has previously marred the use of porosimetry to characterise this material. The results show clear trends in the evolution of the Gilsocarbon graphite porous space. Semi-quantitative deductions are made that will assist the modelling of the evolution of the pore space in the context of the safe extension of the reactors' working lifetimes.
\end{abstract}

\section{Introduction}

The Advanced Gas-cooled Reactors (AGRs) operational throughout the UK use Gilsocarbon graphite as moderator and structural material within their cores and $\mathrm{CO}_{2}$ as a cooling gas. The presence of $\mathrm{CO}_{2}$, under operational conditions, causes Gilsocarbon graphite to undergo radiolytic gasification by activated gaseous species that are present within the open pore structure of graphite [1-3]. These species interact with the walls of the voids within the graphite and subsequently cause the deterioration and mass loss of the solid

\footnotetext{
* Corresponding author.

Email address: glaudone@plymouth.ac.uk (Giuliano M. Laudone)
} 
phase as carbon atoms are gasified to carbon monoxide [4]. Such deterioration adversely affects Gilsocarbon graphite by reducing its structural and mechanical strength [5-7]. Methane is added to the coolant gas stream as an oxidation inhibitor.

Radiolytic oxidation occurs on the external surface of the moderator bricks but more importantly within the internal void space that is accessible to the surface, that is the open pore volume to which the coolant gas can penetrate $[2,8-10]$. Pores which are inaccessible to the coolant gas, and therefore the gasification process, constitute the closed pore volume. Characterisation of the pore structure, and of the evolution of void volumes generated by radiolytic oxidation, should enhance the knowledge and, consequently, improve the modelling of the radiolytic gasification process [4]. Specifically, existing models of Gilsocarbon graphite used and accessed by both EDF Energy and the UK independent graphite advisory committee(GTAC), who advise the office of nuclear regulation (ONR), presume that the radiation-induced oxidation of graphite is dependent on the volume, size and shape of accessible voids, as well as the local gas composition present within the porous structure [11]. The lifetime extension plans for the UK's nuclear reactor fleet depend on the reliability of such models.

Defects introduced to the microstructure by radiolytic oxidation and neutron irradiation are highly complex, and often the effects of each process are studied individually [12-14]. The principal focus for this work was to investigate the impact of radiolytic oxidation on the evolution of graphite microstructure, but it is also necessary to give simultaneous consideration to neutron irradiation damage which introduces point defects into the crystal lattice [15-17]. Many studies have focused on how thermal (which is negligible in AGR design) and radiolytic oxidation affect the structural and mechanical properties of the solid phase of Gilsocarbon graphite [6,15, 18-21], but far fewer have focused solely on characterisation and evolution of the void space [7, 22-24].

This study aims to provide an improved experimental characterisation of the voidage of graphite at various stages of weight loss using a combination of techniques, namely helium pycnometry and mercury porosimetry. The modelling of the void space is the subject of a subsequent publication. 
Bulk densities are calculated from the sample volumes and weights. When combined with helium pycnometry measurements, they yield open and closed pore volumes (OPV and $\mathrm{CPV}$ ) which then reveal trends in the development of the porosity with graphite ageing.

Mercury intrusion porosimetry has previously been shown to be unreliable for the characterisation of virgin nuclear graphite, particularly at the lowest pressures (largest features) [2] and highest pressures (smallest features) [25-27]. Comparative studies between semi-quantitative microscopic interpretations and mercury intrusion porosimetry suggest that porosimetry greatly underestimates the sizes of open macropores when compared with those obtained from image analysis $[2,4]$. A primary cause of the discrepancy is the sensitivity of porosimetry to constrictions in the pore network [28] that cause shielding of larger voids by narrower voids or throats [29].

The shape of the mercury intrusion curve is distinctive in virgin graphites, in that the mercury intrusion does not tail off even at the highest pressure [25-27]. The continuing intrusion at the highest pressures implies a substantial presence of pores below $0.1 \mu \mathrm{m}$. However, repeated measurements on the same specimens show that this region displayed both permanent and reversible damage, as small quantities of mercury were permanently retained by the structure and could not be removed by extended heating at temperature exceeding $300^{\circ} \mathrm{C}$ in vacuo $[26,27]$. The precise mechanism of what was causing the secondary intrusion at the highest applied pressures remains unresolved. Such observations validate the discarding, for modelling purposes, of any mercury intrusion volume which exceeds the void volume measured by helium pycnometry [26]. Investigators of thermally oxidised graphites noted that the excess volume measured by porosimetry was reduced upon thermal oxidation, suggesting that those pores which accommodate the excess intrusion are involved in the thermal oxidation process [1]. Prior to this study, no such observations have been published for radiolytically oxidised graphite samples.

\section{Experimental}

\subsection{Materials}

Both virgin and irradiated Gilsocarbon graphite samples were supplied by EDF Energy Nuclear Generation Ltd, Barnwood, Gloucester, UK. Virgin graph- 
ite specimens were trepanned from spare blocks destined for the Hinkley Point B nuclear reactor, but never deployed. A schematic diagram of the labelling system, which provided the sample identifier for each virgin graphite billet, can be found in the Supplementary Information (SI) Figure 1. Each billet was cut in half to enlarge the data set. A range of irradiated Gilsocarbon material, produced as part of the Blackstone experiment, were supplied for this study. The Blackstone experiment used a Material Test Reactor (MTR) to produce samples that had a radiolytic weight loss and cumulative fast neutron dose which exceeded the conditions observed in current AGRs, thereby creating a material which was nominally similar to end of life AGR graphite.

As the samples had different life histories, they were split into four groups:

- Virgin graphite ; unirradiated Gilsocarbon graphite, as manufactured.

- Irradiated graphite ; of which there are three possibilities:

Ex-virgin, zero weight-loss material that has been irradiated in an inert helium environment during the Blackstone experiment.

Ex-virgin oxidised material that has been subjected to simultaneous irradiation and radiolytic oxidation in capsules containing $\mathrm{CO}_{2}$ as part of the Blackstone experiment.

Ex-AGR material that has been trepanned from working reactors before receiving further oxidation and irradiation dose as part of the Blackstone experiment.

Whilst each batch of Gilsocarbon graphite was originally manufactured to the same specifications for both average density and open pore volume, there remains a large variability between bricks [11]. Variability can still be observed as the sample size gets smaller, due to the natural heterogeneity at every length scale, introducing an additional 'within-brick' variability between neighbouring sub-samples [23]. Ideally, therefore, a very large number of samples should be studied to identify unambiguously the trends masked by the variability. In practice, the number of samples is greatly reduced by the very high cost of preparation, transportation and experimental study of radioactive specimens. In total, 3 different virgin samples and 18 irradiated samples were studied. 
Their cumulative fast neutron dose increments in Equivalent DIDO Nickel Dose $\left(\times 10^{20} \mathrm{n} \mathrm{cm}^{-2} \mathrm{EDN}\right)$ have been converted into their equivalent Displacements Per Atom (dpa) values using a conversion factor of $7.62 \times 10^{20}$ neutrons $\mathrm{cm}^{-2}$ EDN per dpa [30].

\subsection{Sample Preparation}

It is important to avoid artefacts caused by sample preparation, which can cause large differences in intrusion at low pressure. In a previous study [23], it was shown that there was a wide variation between low-pressure mercury intrusion experiments performed on neighbouring sub-samples of the same Gilsocarbon graphite brick. The variation was attributed to surface and edge effects introduced during the milling processes. In that study, the variation in subsamples of a plentiful supply of virgin graphite was reduced, but not eliminated, by machining the samples to cylindrical form using a tungsten carbide tool on a standard lathe, and then cutting across the cylinder with a diamond saw to produce samples of appropriate length. Both techniques ensured ensured smooth, external surfaces.

For this work, all samples had been machined in the same way prior to receipt to minimise artefacts. Virgin samples were provided in cylindrical form, $\sim 11 \mathrm{~mm}$ long and $\sim 5 \mathrm{~mm}$ diameter, whereas all but one of the irradiated samples was smaller, measuring $\sim 6 \mathrm{~mm}$ long and $\sim 5 \mathrm{~mm}$ diameter.

All specimens were washed with isopropanol in an ultrasonic bath, until no dust was visible in the solution. The samples were then dried for a minimum of 3 hours at $305{ }^{\circ} \mathrm{C}$ using the BELPREP-vac (MicrotracBEL, Japan). Drying was repeated between each experimental measurement to ensure surfaces were free from contamination.

\subsection{Density measurements}

Two sets of dimensional measurements were provided for each irradiated sample: one set prior to entering the Blackstone experiments (as trepanned values) and a second set measured after further irradiation. The latter values were used to calculate bulk volume $\left(V_{\mathrm{BULK}}\right)$, and with the mass $(m)$ of the samples were used to calculate values of bulk density $(\rho)$. 


\subsubsection{Micropycnometry}

Skeletal density $\left(\rho_{\mathrm{s}}\right)$ measurements were obtained using a specially constructed helium micropycnometer, shown in the SI Figure 2. Accurate measurements on the small samples were made possible by minimising the dead volume in the sample and reference chambers and use of a very high precision and accuracy pressure transducer. Measurements were performed in triplicate at room temperature and an average calculated for each sample. A new blank run was performed for each of the triplicate runs to account for any fluctuations in ambient temperature.

The volume of the solid phase $V_{\text {SOLID }}$ was calculated by assuming a theoretical density of an ideal (non-porous) graphite crystal density of $2.26 \mathrm{~g} \mathrm{~cm}^{-3}$. The CPV and OPV for each sample, which represents the volume ratio of open and closed porosity present throughout the sample, were calculated using equations 1 and 2 respectively:

$$
\begin{gathered}
\mathrm{CPV}=\frac{m-V_{\text {SOLID }} \times \rho_{\mathrm{s}}}{\rho_{\mathrm{s}}} \\
\mathrm{OPV}=V_{\text {BULK }}-\mathrm{CPV}-V_{\text {SOLID }}
\end{gathered}
$$

\subsection{Mercury Intrusion Porosimetry (MIP)}

The samples were analysed using the PASCAL 140 and PASCAL 440 mercury porosimeters (Thermo Fisher Scientific, Milan), which perform the analysis at low $(\leq 400 \mathrm{kPa})$ and high $(\leq 400 \mathrm{MPa})$ pressure ranges respectively. The instruments were operated in PASCAL mode which allowed the mercury pressure to re-equilibrate at each value of applied pressure when intrusion or extrusion was detected.

All mercury intrusion porosimetry curves were corrected with a blank run with an empty sample chamber, which compensates for sample chamber defects and mercury compression. Traditional practice is to interpret the percolation data using the Laplace equation, Equation 3, which relates the pressure $(P)$ of a non-wetting fluid with surface tension $(\gamma)$ and contact angle $(\theta)$ to the diameter of a cylindrical pore-entrance diameter [31]:

$$
d \geqslant \frac{-4 \gamma \cos \theta}{P_{\mathrm{app}}}
$$


A value of $140^{\circ}$ was used for the advancing (intruding) contact angle, and $130^{\circ}$ for the receding contact angle [32]. Employing such values as constants assumes that the nature of the surfaces of the sample does not change during the process of intrusion and extrusion [23]. A value of $0.480 \mathrm{~N} \mathrm{~m}^{-1}$ was assumed for the surface tension of mercury.

\section{Results}

\subsection{Density measurements}

The results from the micropycnometry experiments are listed in Table 1 and shown in Figures $1-2$. The table shows the average results and instrumental relative standard deviations (RSDs) for the triplicate measurements. The bulk density measurements for virgin Gilsocarbon samples are in good agreement with those provided by the manufacturer (SI Table 1) and previous measurements by Laudone et al. [23].

Only the instrumental repeatability has been taken into account in the error bars shown in Figure 1. This is $\pm 3.5 \%$ at worst, and good enough not to mask the trends between samples. However, measurements for the calculated weight loss and cumulative fast neutron dose, particularity for ex-AGR material, have additional uncertainties mainly arising from the heterogeneity of the virgin material. The typical error associated with weight loss for ex-AGR material is $\sim 2 \%$ (absolute), whereas in ex-virgin material this value is experimentally measured. The errors for cumulative fast neutron dose are more complicated to estimate; for the Blackstone experiment samples, the relative uncertainties are quoted as 10\%, and comparable errors are possible for ex-AGR samples. These various errors and uncertainties must be borne in mind when comparing samples, particularly because ex-virgin and ex-AGR samples are subject to different types of error.

Figure 1a shows that, whilst the results of the skeletal density measurements are somewhat scattered, they tend towards a higher value of skeletal density with increased weight loss. This could only be the case if previously inaccessible volume was being opened, presumably due to oxidative processes. Therefore, for each sample, the corresponding closed and open pore volume have been calculated using equations 1 and 2 respectively, assuming the density of the nonporous solid phase to be equal to the theoretical crystal density of graphite. As 
Table 1: Summary of nuclear graphite density values derived from helium pycnometry, and porosity values derived from pycnometry and mercury porosimetry, showing instrumental relative standard deviations (RSDs). The average envelope density was calculated geometrically and average skeletal density by helium pycnometry. All values have been rounded to 2 decimal places.

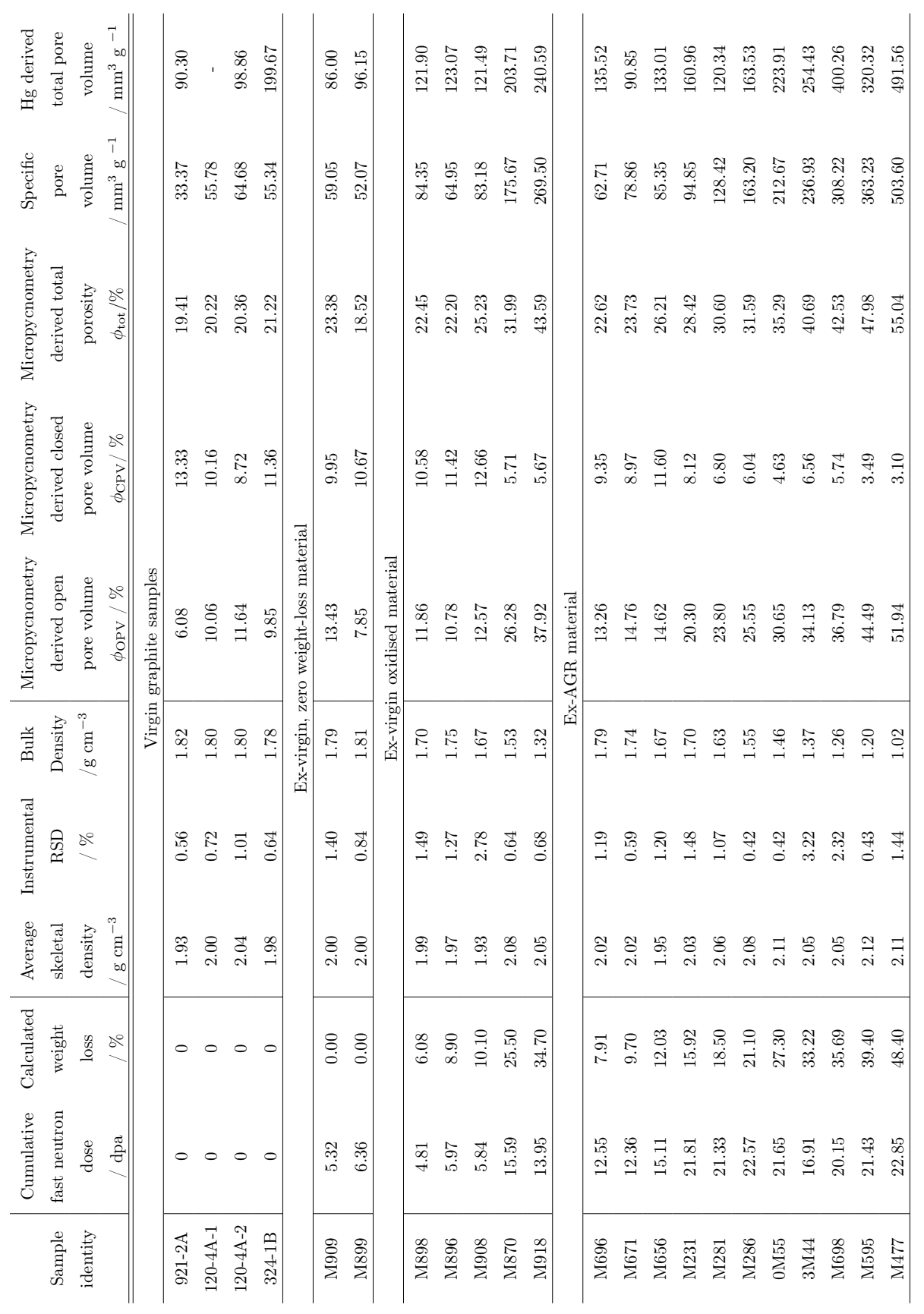




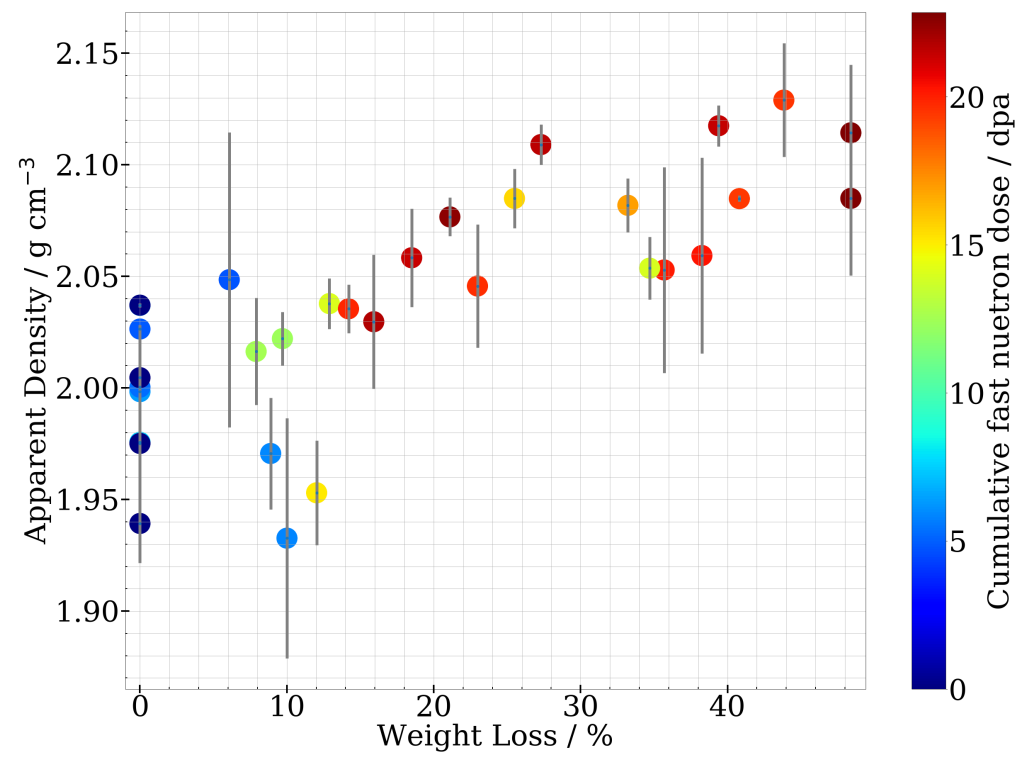

(a)

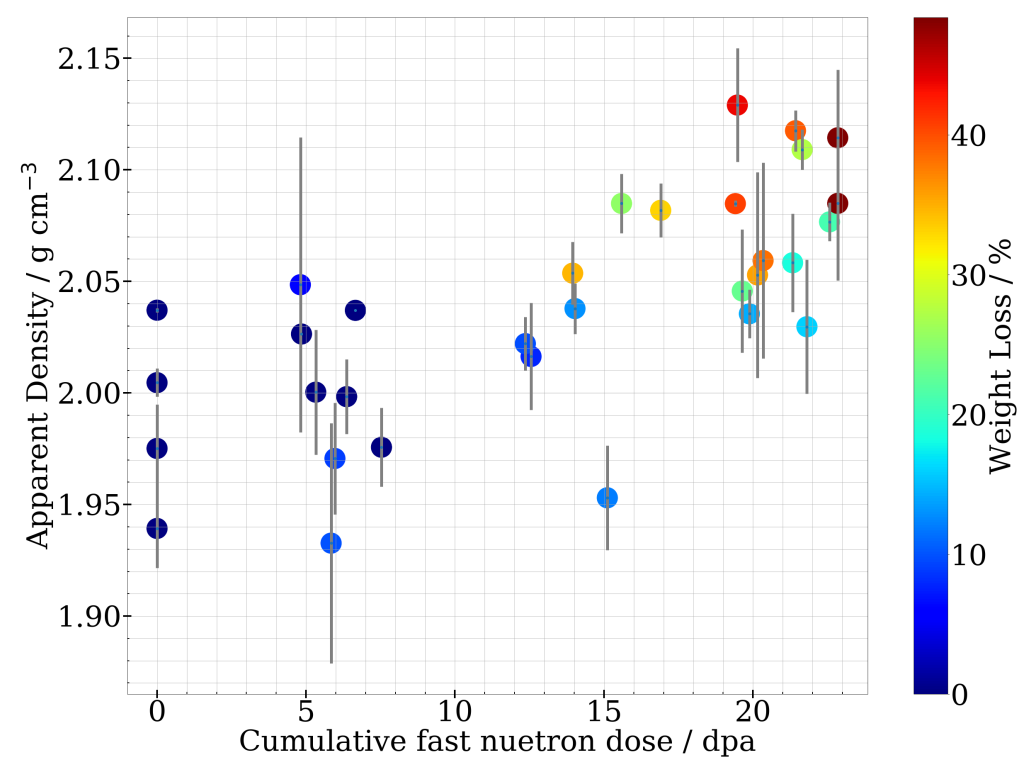

(b)

Figure 1: Gilsocarbon graphite skeletal densities $\rho_{\mathrm{S}}$ versus (a) calculated weight loss and (b) calculated cumulative fast neutron dose, with error bars showing instrumental repeatability. 
expected, the results were all lower than the theoretical crystal density of 2.26 $\mathrm{g} \mathrm{cm}^{-3}$. Figure $1 \mathrm{~b}$ highlights the variability of the virgin (zero dose) material, shown in dark blue, in the range $1.99 \pm 0.05 \mathrm{~g} \mathrm{~cm}^{3}$.

It can be seen in Figure 2 that, for virgin material, the porosity is roughly equally divided between that which is accessible to coolant gas, OPV, and that which is isolated, CPV, indicated by the symbols on the graph at zero weight loss and/or zero dose, some of which are superimposed. Consequently, the mass loss, caused by radiolytic oxidation, predominately impacted the evolution of the OPV, which increased linearly throughout the weight loss series. The linear reduction of the CPV for the weight loss series is minor in comparison and does not reach zero. This is in agreement with data collected on other grades of nuclear graphite, which showed comparable evolution of the pore volumes [33]. Similar behaviour can also be identified in relation to cumulative fast neutron dose (Figure 1b). However, the trends are more scattered, partly because samples with similar weight loss trend differently with cumulative fast neutron dose (shown by similarly coloured points on the graph).

Consideration of specific results in Table 1 reveals the extent of the sample variability mentioned above. Samples $120-4 \mathrm{~A}$ and $324-1 \mathrm{~B}$ were cut from the same billets as M899 and M909 respectively. For both 120-4A replicates the total derived porosity (20.22\% and $20.36 \%$ ) was larger than that observed for sample M899 (18.52\%). Whilst the OPV was larger in the virgin material $(10.06 \%$ and $11.64 \%$ compared with $7.85 \%$ ), the CPV was larger in the irradiated specimen (10.67\% compared to $10.16 \%$ and $8.72 \%$ ). For sample $324-1 \mathrm{~B}$ the total porosity $(21.22 \%)$ and OPV $(9.85 \%)$ was smaller than in the irradiated specimen (23.38\% and $13.43 \%$ respectively), and the reverse relationship was observed for CPV (11.36\% for 324-1B and $9.95 \%$ for M909). Thus the intrabillet variability present in the virgin material masked any observable trends that may have developed upon irradiation.

Sample 921-2A (virgin) was cut from the same billet as M896 (ex-virgin oxidised). During graphite ageing, the bulk density of the sample was lower in the oxidised material $\left(1.75 \mathrm{~g} \mathrm{~cm}^{-3}\right)$ when compared with its virgin counterpart $\left(1.82 \mathrm{~g} \mathrm{~cm}^{-3}\right)$, which corresponded to a more porous medium. The CPV was also lower in M896 (11.42\%) than in the virgin material (13.33\%), and consequently 


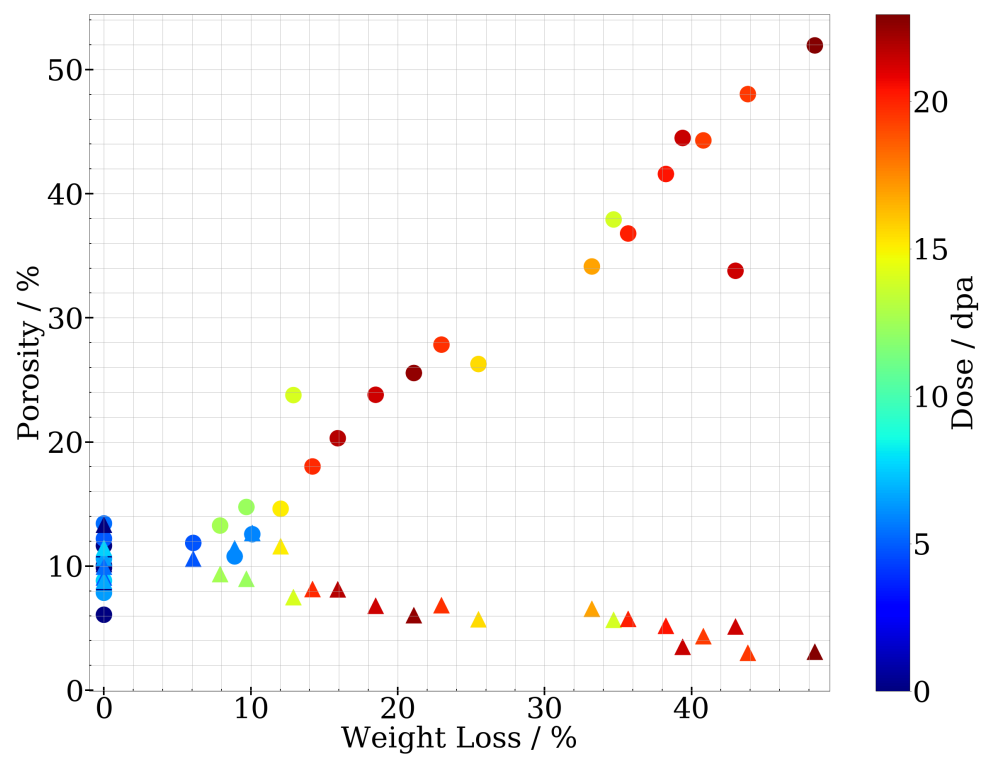

(a)

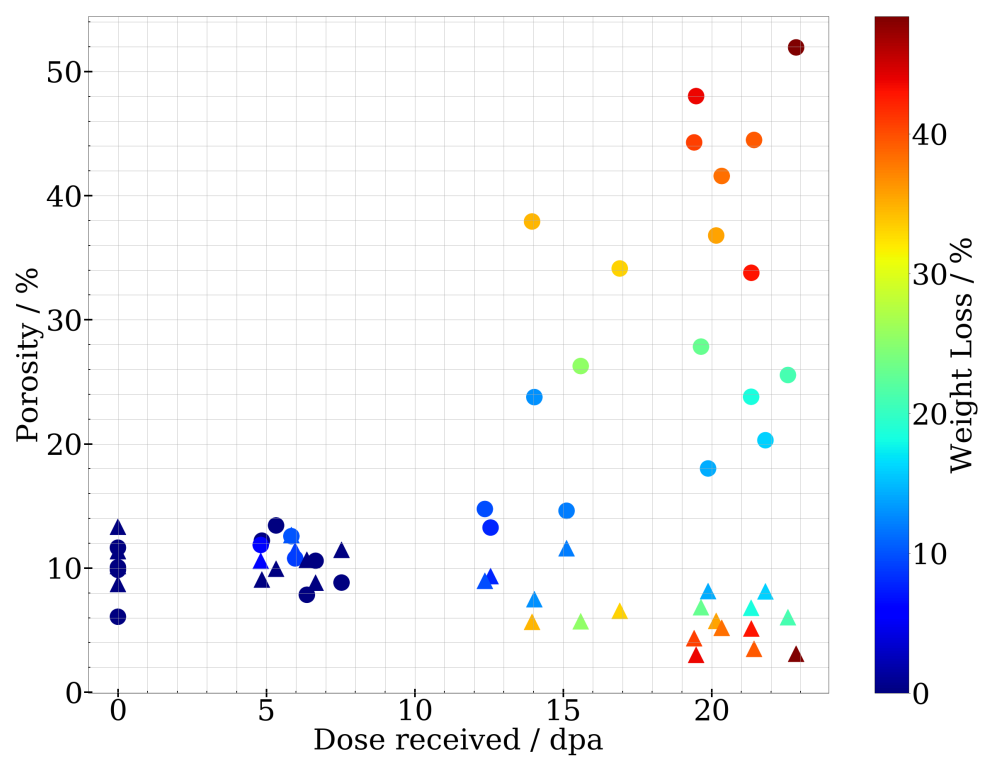

(b)

Figure 2: Calculated OPV (circles) and CPV (triangles) values for all Gilsocarbon graphite samples versus (a) calculated weight loss and (b) cumulative fast neutron dose 
the skeletal density was larger in the oxidised sample $\left(1.97 \mathrm{~g} \mathrm{~cm}^{-3}\right.$ for M896 relative to $1.93 \mathrm{~g} \mathrm{~cm}^{-3}$ for sample $921-2 \mathrm{~A}$ ). The total and OPV were larger in the oxidised specimen (22.20\% total porosity and $10.78 \%$ OPV) when compared with the virgin material ( $19.41 \%$ total porosity and $6.08 \%$ OPV). These findings are in agreement with the overall trends expressed in Figure 2.

\subsection{Mercury intrusion porosimetry (MIP)}

A selection of mercury porosimetry curves for the different groups of samples is now presented. Those individual percolation curves not included in the main body of the text can be found in the Supplementary Information, Figures 35. The total intruded volumes accessed by mercury are tabulated in Table 1. Due to instrumental issues it was not possible to collect mercury extrusion data for one ex-virgin zero weight-loss sample, M899, one ex-virgin oxidised sample, M918, and one ex-AGR specimen, M231. For these samples, only the intrusion curve is available.

\subsubsection{Virgin Graphite}

Mercury percolation curves for the three virgin samples are shown in Figure 3. Relative to the overall wide range of total intrusion and percolation characteristics curves presented below, these are relatively similar to each other. The variation is representative of the inter-sample variability mentioned previously.

Different intrusion behaviours were observed at low pressures between the virgin samples: 921-2A shows a sigmoidal intrusion curve whilst the other two virgin specimens show less stepped intrusion from the onset of pressure. All three virgin specimens show intrusion after the percolation curve plateaus, which we refer to as 'secondary intrusion', which continues to rise even at the highest operating pressures. The uniaxial compressive strength for virgin Gilsocarbon graphite has previously been measured at 74.6 MPa with the grain and $74.7 \mathrm{MPa}$ against the grain [34], represented in Figure 3 by the green dashed lines. The point at which the second volume increase occurred for samples 921-2A and 120-4A, Figures $3 \mathrm{a}$ and 3b, corresponded closely to the quoted values, and strongly suggests that this intrusion was attributable to sample compressibility and damage to the internal void structure [26]. This deduction is supported by the lower maximum helium accessible volumes per gram of sample, measured by micropycnometry, which are displayed as a blue dashed lines on 


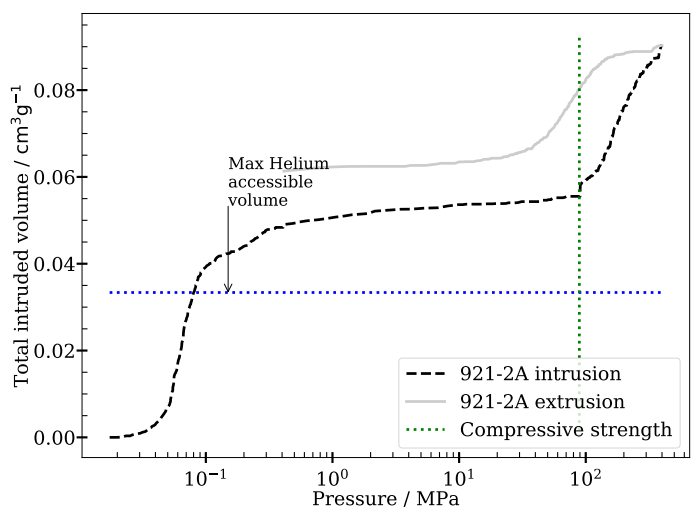

(a)

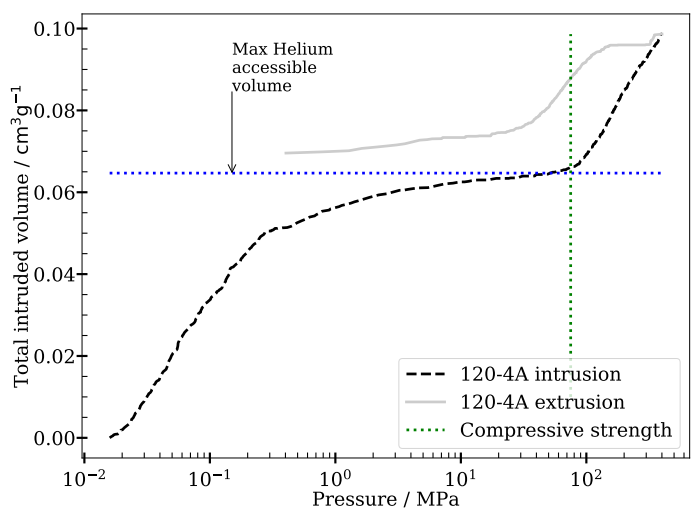

(b)

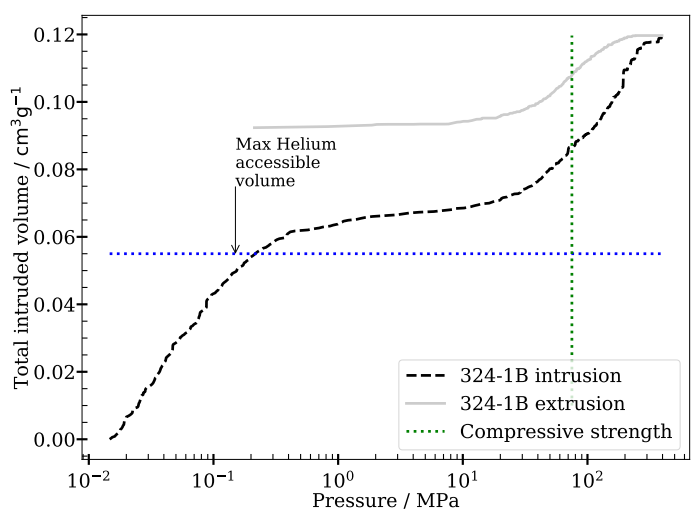

(c)

Figure 3: Cumulative mercury intrusion and extrusion curve for the virgin graphite samples: (a) 921-2A; (b) 120-4A and (c) 324-1B. Also shown on the graph is the maximum helium accessible volume obtained via micropycnometry (blue dashed line) and the uniaxial compressive strength of virgin Gilsocarbon (green dashed line [34]). Cumulative MIP percolation curve(a) 921-2A-1 (b) cumulative fast neutron dose 
the individual percolation curves. Additionally, upon depressurisation of the mercury leading to secondary imbibition of a nominal air or vacuum phase, 20$30 \%$ of the previously intruded mercury exited the void structures by capillary expulsion forces, whilst the majority of the mercury remained trapped. The onset of secondary intrusion in sample 324-1B occurred at applied pressures lower than the other two virgin samples $(\sim 20 \mathrm{MPa})$, and before the quoted value of compressive strength. The operating conditions for mercury intrusion porosimetry could be responsible for this result as, in contrast to the uniaxial compressive strength measurement, pressure is applied to the sample triaxially.

\subsubsection{Ex-virgin, zero weight-loss material}

Figure 4 shows the percolation curve for two graphite samples within this group of materials. The percolation curves show similar intrusion, both at high and low pressure. Additionally, both specimens display similar total intruded volumes which are comparable to those obtained for the virgin specimens. This is highlighted in the individual plots, shown in Figure 3 SI, where the percolation curves for the corresponding virgin graphites, cut from the same billets (120-4A and M899, and samples 324-1B and M909), are presented on the same graph. Whilst the inherent variability in the virgin graphite material masked any visible trends when comparing values of skeletal density, some comparative information can be obtained by overlapping the percolation curves and these are discussed in more detail below. 


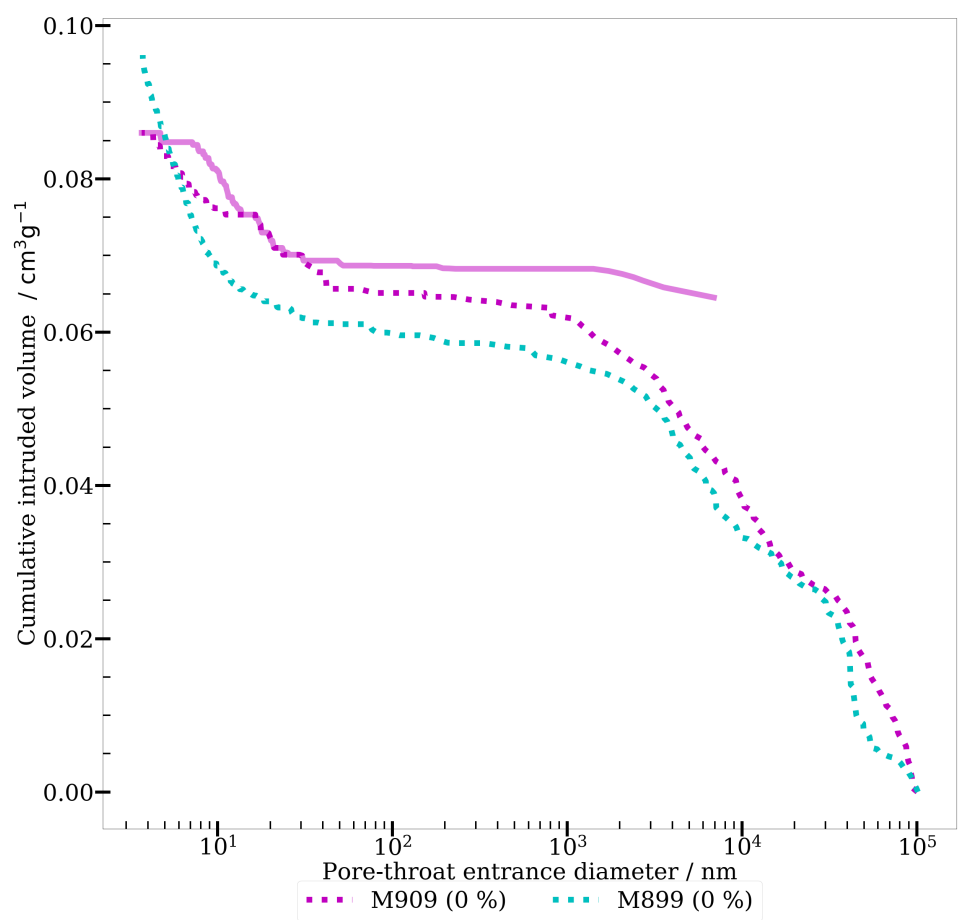

Figure 4: Cumulative mercury intrusion (dotted) and extrusion (solid) for Ex-virgin, zero weight-loss material plotted against their nominal pore-throat Laplace diameters. Weight loss shown in brackets. 


\subsubsection{Ex-virgin oxidised material}

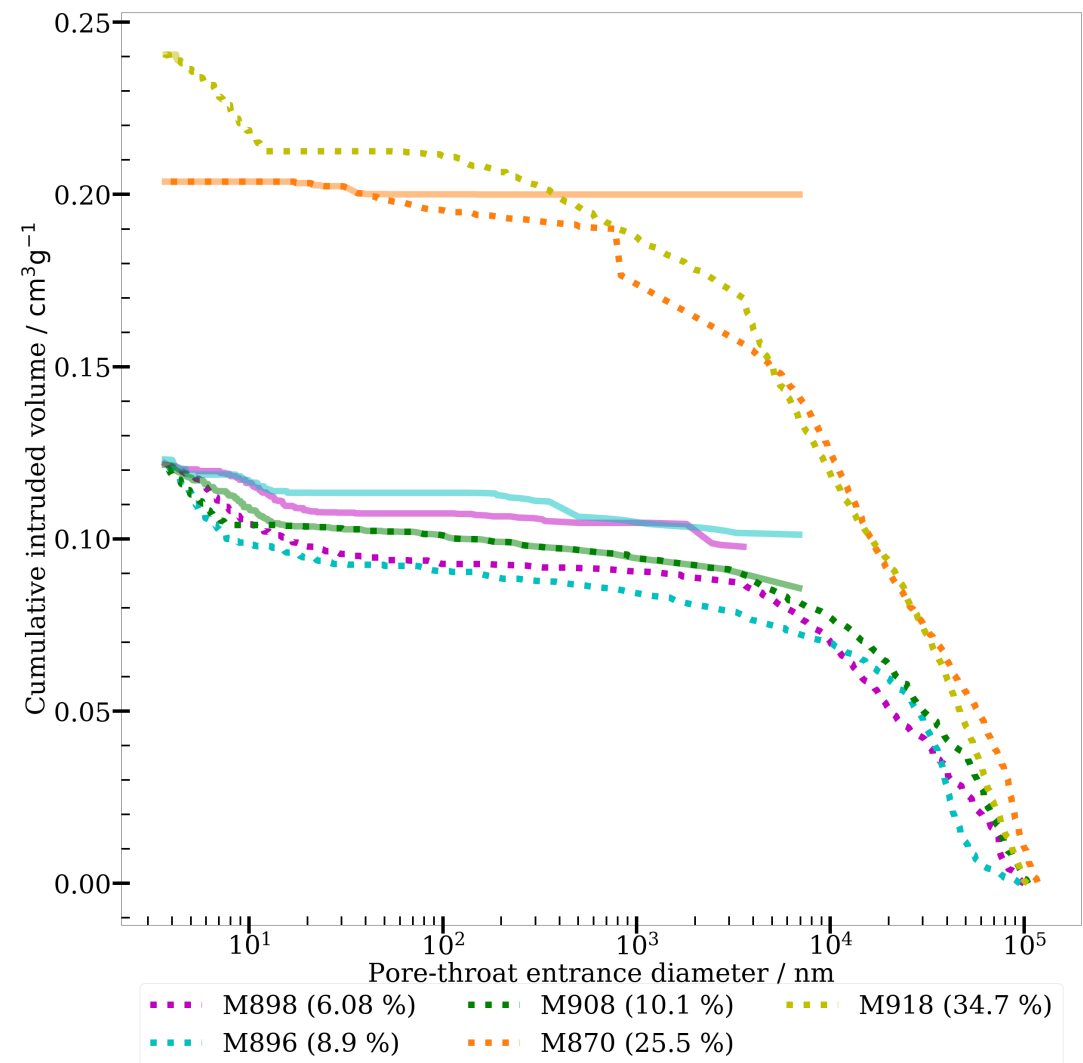

Figure 5: Cumulative mercury intrusion (dotted) and extrusion (solid) for ex-virgin oxidised material plotted against their nominal pore-throat Laplace diameters. Weight loss shown in brackets.

Figure 5 shows that samples M898, M896 and M908 displayed similar intrusion curves that asymptote towards a comparable total volume around 0.12 $\mathrm{cm}^{3} \mathrm{~g}^{-1}$. The cumulative fast neutron dose and calculated weight loss for each of these samples were closely comparable; it is therefore not unreasonable to expect these samples to have similar percolation curves. Sample M896 was part of the same manufacturing heat batch as the virgin samples 921-2A-1 so, again, the corresponding virgin graphite data has been overlaid on the individual graph for M896 (visible in Figure 4 SI) in order to identify any evolutionary trends. Sample M870 did not display secondary intrusion but did show a sharp increase in intrusion at a pressure of $0.3 \mathrm{MPa}$. This phenomenon is discussed in more detail in the discussion section. 


\subsubsection{Ex-AGR material}

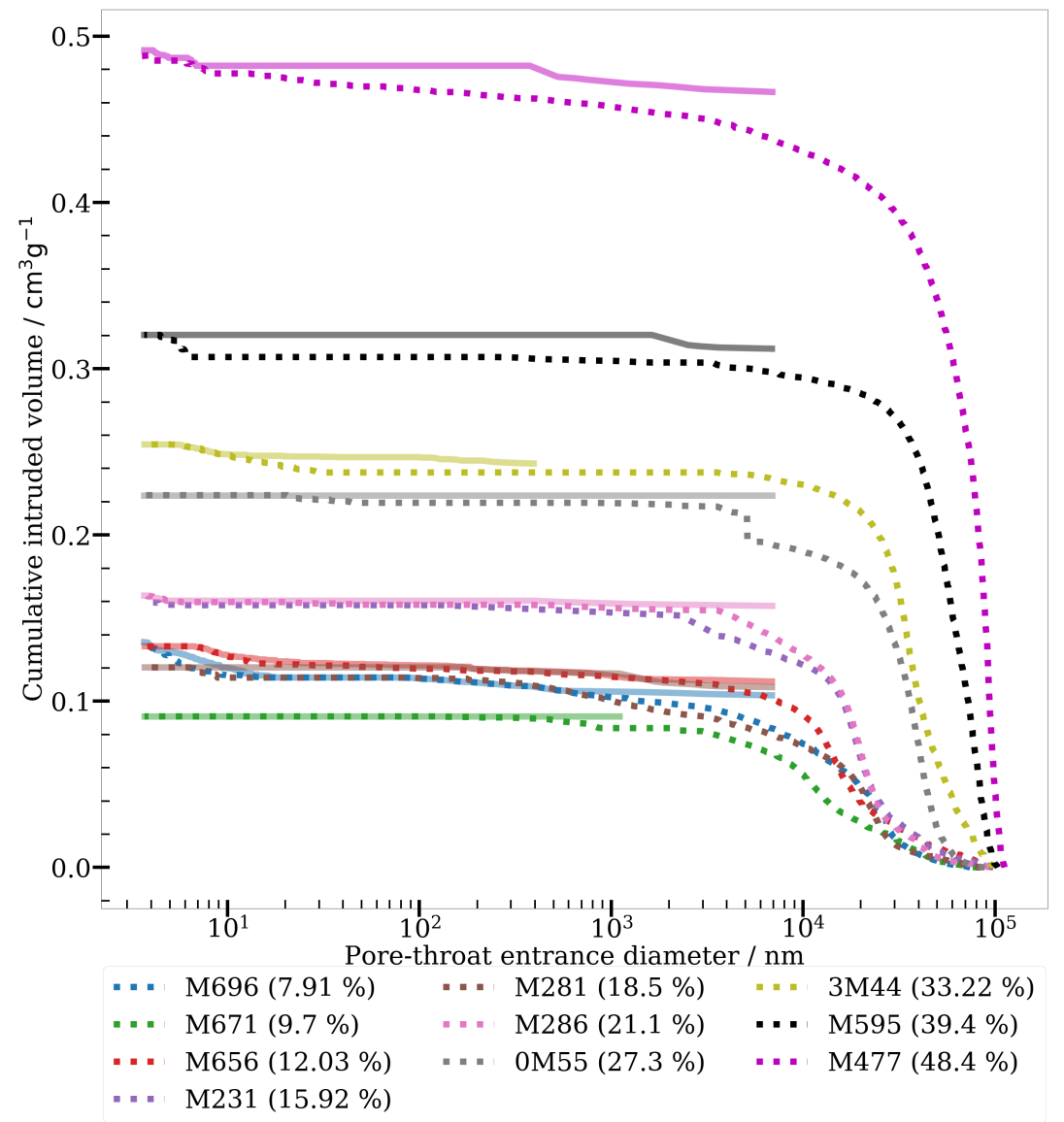

Figure 6: Cumulative mercury percolation curves for ex-AGR samples plotted against their nominal pore-throat Laplace diameters.

For the ex-AGR series, three dominant trends can be identified. Firstly, it can be seen both from Figure 6 and the values in Table 1 that the lower weight loss series comprised a lower total intruded volume, whilst increased mass loss resulted in a higher total intruded volume, as would be expected. Secondly, there is a notable change in the shape of the intrusion curve with increased weight loss. As the mass loss within the samples increases, there is progressively increased intrusion corresponding to pore-throat entrance diameters above $2000 \mathrm{~nm}$, and the sigmoidal intrusion, observed at low pressure, becomes increasingly linear: the oxidation induced appearance of features larger than those the instrument can identify, results in disappearance of the initial gradual mercury intrusion. Lastly, with progressive mass loss, samples show more comparable values of 
helium and mercury accessible volumes, Table 1 . This has been highlighted on the individual graphs which can be found in the Supplementary Information, Figure 5. This is in accord with the reduced secondary intrusion seen at higher pressures. A summary plot of the maximum cumulative volumes with weight loss can be visualised for all samples in Figure 6, provided in the Supplementary Information.

\section{Discussion}

\subsection{Pycnometry}

Figure 1 shows a general increase in skeletal density across the entire weight loss series. The observable range in which the virgin graphite void space can evolve is very narrow, due to the small contribution of CPV in the virgin material, around $10 \%$ of the total sample volume. If all of this CPV was to be removed, there would only be a small increase in skeletal density, of the order of $0.2-0.4 \mathrm{~g} \mathrm{~cm}^{3}$ for most virgin graphites.

Figure 2 presented the micropycnometry data as OPV and CPV contributions. It has been proposed by Hacker [35] that OPV and CPV are evenly distributed in virgin Gilsocarbon graphite, and this is confirmed by the overlapping OPV and CPV points at zero weight loss in Figure 2a. However this relationship changes once the samples are subjected to oxidation. The OPV increases almost linearly with weight loss and dominates the total porosity contribution The same trend was observed with respect to increasing cumulative fast neutron dose, Figure 2b. The latter results are much more scattered, although it can be seen that there are subsidiary trends with samples of similar weight loss. For example the increase in OPV with cumulative fast neutron dose is much less marked for samples with low weight loss $(\bullet)$. Some of the scatter in Figure $2 \mathrm{~b}$ may be due to the uncertainties in cumulative fast neutron dose, section 3.1. However, it is also known that weight-loss evolves much more quickly with ionising-radiation dose (principally gamma radiation emitted from the reactor fuel, but also, to a lesser extent, from the unstable carbon-atom nucleus after collisions with fast neutrons [36]) which could explain the disparities between trends observed in fig and. For both graphs in Figure 2, the OPV increases much more than the CPV decreases, showing that the OPV is not simply created making CPV accessible. 
Micrographs taken for virgin Gilsocarbon graphite samples impregnated with a fluorescent dye have revealed that much of the initial CPV resides in the layered, onion-skin-like structure within the filler particles [8]. Other CPV contributions are contained in the pitch-binder phase in the form of cracks and trapped gas bubbles formed during the manufacturing process [2].

There have been several studies on the evolution of CPV with irradiation. Firstly accommodation cracks, in the form of the preferentially aligned graphitisation cracks that develop parallel to the basal planes, close due to c-axial expansion of the graphite crystals [20]. This occurs before 'turnaround' when all of the accommodation porosity is closed through c-axial expansion, at which point the bulk graphite begins to grow under continuing irradiation. Turnaround can be delayed with respect to ionising-radiation dose by the expansion of accessible thermal shrinkage cracks via radiolytic processes which increases the amount of accommodation porosity [37].

Other workers have proposed that the closed porosity in the Gilsocarbon filler particles opens up in the early stages of oxidation [2]. Such studies used quantitative image analysis to show that radiolytic oxidation caused the open pore volume in the binder phase to interconnect with some of the previously closed porosity contained in the filler particles [4]. However, these investigations were performed on ex-virgin, oxidised material under MTR conditions. Such pore space will not participate in radiolytic oxidation under AGR conditions until they are made accessible to the circulating $\mathrm{CO}_{2}$ by gas pressure, or by progressive mass loss of the surrounding solid phase [23]. A recent study by Shen et al. [38] identified the presence of a unique structural boundary that encapsulated the Gilsonite coke filler particles in some virgin specimens. Optical light micrographs revealed this barrier, otherwise referred to as Binder-1, as a bright ring around the filler particles which is indicative of compact, dense crystallites. Raman analysis also found the domain size of the Binder-1 crystallites to be roughly an order of magnitude larger than that of the Gilsonite coke filler particle. Latest theories propose that, under AGR conditions, throughout early life, irradiation damage causes the Binder-1 crystallites to become more dense and orientated around the majority of the Gilsocarbon filler particles to form a "mosaic" boundary [9]. One hypothesis is that this layer provides an initial 
protective barrier against the oxidative species, preventing breakthrough into the filler particle. Simultaneously, irradiation damage is driving the dimensional change of the crystallites within the filler particles, closing internal porosity. This, in turn, reduces the CPV whilst thickening the protective layer. It has been hypothesised that under typical AGR conditions, the consolidation of this barrier occurs faster than the rate at which oxidation can erode the coating, thereby reducing the rate in which the Gilsocarbon filler particles are broken into. This is in agreement with the results shown in Figure 2 where the CPV decreases gradually and linearly with increasing weight loss, but does not fall to zero, suggesting that even in the highest weight loss sample, a portion of the porosity remains inaccessible to the coolant gas. Current proposals are that, for ex-AGR material, oxidative opening of CPV saturates at relatively low ionisingradiation dose (5 dpa) [36]. A possible explanation for this could be that beyond the saturation fluence, oxidation no longer affects the development of CPV due to the development of the mosaic barrier. Looking at Figure 2a, the continued trend at higher values of cumulative fast neutron dose may suggest that this barrier is only effective up until a certain weight loss, and therefore the current models may need to be re-evaluated. However, further measurements on a wider range of AGR samples would need to be obtained in order to support or reject these assumptions definitively.

\subsection{Mercury porosimetry}

When making inferences from the shapes and magnitudes of mercury porosimetry measurements, it is essential to avoid the fallacy of assuming that the slope of the intrusion curve gives direct information about the sizes of the intruded voids [29]. However, some valid deductions are possible.

\subsubsection{Low pressure intrusion characteristic}

Two distinct slopes were observed in the low pressure intrusion curves of virgin graphite (Figure 3). Sample 921-2A displayed a sigmoidal distribution at the lowest pressures which is typical of most porous materials. This implies that the material comprised a non-hierarchical network with similarly sized constraining pore-throat entrances, which, once the corresponding pressure was overcome, provided access to the majority of the void system and filled with mercury accordingly. By contrast, the initial percolation curves obtained for 120-4A 
and 324-2B at low pressure revealed a more linear increase in intrusion over a similar pressure range, suggesting the presence of a hierarchical network with void-throat entrances in the approximate range of 2 to $100 \mu \mathrm{m}$. A hierarchical network is one in which voids of different sizes can be grouped by size, and the groups assumed to have separate, independent properties - characterised using, for example, 'two-pore' or 'dual pore' models.

The ex-virgin, oxidised specimens allowed increased intrusion at low to medium pressure, when compared to the virgin or ex-virgin, zero weight-loss material. A comparison of the virgin specimen M921-2A and the oxidised sample M896 demonstrates that radiolytic oxidation increased the overall porosity of the sample, with the increase appearing quite uniformly over the entire range of intrusion pressure. This is in agreement with the reported observation that, at low weight loss, erosion of the void system is uniform throughout the pore structure [2].

It can also be observed that, for samples past $35 \%$ weight loss, the percolation curves have shifted towards the right (i.e. larger pore-throat entrances dominate the intrusion curve). This trend progressed throughout the higher weight loss series, where the highest mass loss specimens verged towards vertical intrusion as the largest pore-throat entrances surpassed the measurable upper limit $(>115 \mu \mathrm{m})$ of the instrument. Such large features supports the idea of pore coalescence; up to $35 \%$ weight loss, the void network was uniformly developed by the gasification process, enlarging existing pores; beyond this threshold neighbouring pores begin to coalesce creating large voids that dominate the intrusion paths. Larger, interconnected open void networks were also observable in the high weight loss porosimetry curves, such as M595, Figure 6 .

\subsubsection{Overall intrusion volume and high pressure intrusion characteristic}

When comparing the total accessible void volumes obtained for the entire series of samples, it was observed that at zero or low mass loss, larger values of total accessible volume were achieved from mercury porosimetry than observed with micropycnometry (Figure 3 and Figures $3-5$ in the Supplementary Information). The discrepancy reduced with increased mass loss, up to $20 \%$ mass loss at which the samples showed values in relatively close agreement. Further increases in weight loss resulted in helium accessible volumes that were larger 
than the total void volume measured by porosimetry. The disparity between total intruded mercury volumes and helium accessible volumes indicates that at higher pressures, mercury is not simply intruding voids - it is damaging the sample and also causing elastic compression. Damage seems to dominate, so will be discussed first.

The first clue with regards to sample damage is to compare virgin specimens with ex-virgin oxidised and ex-AGR specimens. All three virgin graphite samples showed secondary intrusion at pressures above the material's compressive strength value (indicated by the green dashed lines in Figure 3), as previously reported by other workers [23, 25-27]. With the increasing mass loss that occurs in the ex-virgin oxidised and ex-AGR specimens, the extent of secondary intrusion at the highest pressures is reduced, but does not disappear entirely. This, together with the more compatible total accessible void volumes measured by micropycnometry, indicate that the void features altered by radiolytic oxidation are those involved in the processes which gave rise to the secondary intrusion and the corresponding damage or compression of the sample.

When graphitic structures are stressed under high pressures, the crystalittes can slip or reorientate, deforming the microstructure. This deformation is reversible upon reduction of the applied pressure [39]. This process, if present, could result in the opening and closure of inaccessible voids at the highest pressures, thus influencing the mercury intrusion and extrusion characteristics. If the secondary intrusion observed at high pressure was predominately caused by the opening of previously inaccessible porosity, it could be postulated that there would be less secondary intrusion in oxidised samples with lower CPV. Such a trend was observed, as discussed below. The quantitative basis of the postulate is supported by the observation that for all samples, the volume of $\mathrm{CPV}$ present was larger per gram of sample than the volume intruded during secondary intrusion.

Another proposal is that, additionally to graphite 'twinning', such intrusion may also result from a 'bridging' phenomena presented in electron micrographs by Liu et al. [40], where solid phase features inside the internal void network snap and rearrange forming bridges across void channels. The existence of bridging in virgin graphite, which causes a reduction in the void entrance diameters, could 
explain both secondary intrusion at high pressure (small void entrances) as well as the trapping of mercury within the void structure upon depressurisation [26]. Upon oxidation it would be expected that such features present within the flow channels would be eroded and, hence, reduce such effects.

Other published suggestions are based on the complexity of the microstructure formed during the manufacturing process [23]. Impregnation of coal tar pitch, used to increase the density of Gilsocarbon during manufacture, may have caused entrances into individual pores, or into groups of interconnected pores, to have become partially or fully blocked upon graphitisation, thus causing an increase in applied mercury pressure necessary to intrude them. Such blocking would also cause trapping of mercury upon depressurisation, which has been observed in cyclic porosimetry at every intrusion pressure [23] and for all samples in this study, but to a lesser extent in the ex-AGR oxidised samples.

As well as sample damage, one must also consider elastic compression at pressures below the compressive strength of the material. Elastic compression is indicated by the overlap of the extrusion curve with the intrusion curve as the pressure is released and the sample re-expands [26], which was observed for the oxidised samples but not for the virgin specimens. Since the solid phase material is the same, the inference must be that the virgin samples are damaged at lower intrusion pressures than oxidised samples, and that the damage masks any elastic compression in the virgin samples. A likely explanation is that in an oxidised sample, the connectivity of the void network increases and therefore is able to fill with mercury before the highest pressures are reached. Upon further increases in pressure the mercury-filled pores provide some rigidity against the compressive forces and prevent fracture. This is in contrast to a virgin or low weight loss material, where some networks will be hidden behind smaller entrances and consequently will not be filled prior to the onset of the high pressures. Without the support of mercury filled pores, the graphite can shear and break under local pressure differentials larger than can be withstood by the compressive strength of the solid phase. This is supported by a previous study which demonstrated that the moderating virgin graphite could be pressurised up to $100 \mathrm{MPa}$ in ethanol without causing any damage to the structure [2]. Alcohol, in contrast to mercury, is a wetting liquid, so will have permeated into all the 
features before pressure was applied, ensuring there is no differential pressure across voids. By contrast mercury, as a non-wetting fluid, will only enter a void system when a threshold value of pressure is applied. It should be noted that the same pressurisation experiments in ethanol were performed on a reflector grade of graphite (SM1-24) in which damage did occur [2]. However, the shape of the intrusion curves where damage was observed showed a uniform expansion of the pore volume over the entire pressure range which, when considering the material characteristics of SM1-24, can be explained by its increased total porosity and, consequently, lower compressive strength that Gilsocarbon.

Another suggestion relates back to the manufacture of the Gilsocarbon graphite. Gas evolution pores, which form in the binder phase during the baking and initially reside as closed pores, may migrate vertically towards the surface of the graphite block during cooling. This will create a series of narrow gas vents or thin pore walls which, with increasing applied pressures, could be broken into [23, 25].

Other forms of CPV, such as Mrozowski cracks, are present in both filler particles and the binder phase. If forced open at the highest pressures, it is possible that they could close upon relaxation during depressurisation, permanently retaining mercury inside the structure; this would explain the permanent retention of mercury observed in other grades of nuclear graphite even upon prolonged periods of heating under vacuum [26]. However, these cracks provide accommodation porosity which close over time due to crystal expansion during irradiation. It could therefore be expected that after turnaround a reduction in the excess intrusion would be observed, due to the lower presence of accommodating CPV. The extrusion curves observed for the entire series (Figures 3 6) reflect such behaviour.

It has been previously observed that the secondary intrusion also decreases as a result of the thermal oxidation of Gilsocarbon [1]. Thermal oxidation is much simpler to study than radiolytic oxidation, but the structural change mechanisms are so different that the results offer no additional insights for the present study. 


\subsubsection{Zero weight loss specimens}

Closer inspection of the differences between individual samples can also provide useful inferences, although these are more speculative because of the endemic heterogeneity between samples.

The ex-virgin, zero weight-loss specimens both displayed similar intrusion to that observed for the virgin samples. When comparing these samples to their virgin counterparts, both samples displayed reduced total cumulative intruded volumes, although this was more evident for sample M909. By overlapping the curves, it can be seen in Figure 3 SI, that the reduction of intruded mercury was experienced over the entire percolation curve. This could allude to the bulk shrinkage of graphite, experienced upon the introduction to an irradiative environment, pre-turnaround, affecting the pore-throat entrance diameters uniformly over the entire microstructure. Additionally, the reduced intrusion at the highest pressures suggested that the mechanisms involved in accommodating the secondary intrusion were affected by the presence of irradiation and ultimately contributed to a lower total intruded volume. The extent to which the secondary intrusion occurred at the highest pressure was greatly reduced for sample M909, but the changes were less obvious in sample M899. However, the initial sample variability in the virgin material may conceal explicit trends.

\subsubsection{Ex-virgin, oxidised specimens}

Previous research on ex-virgin oxidised material showed that the closed porosity in the Gilsocarbon filler particles opened up in the early stages of corrosion [2]. It is proposed that the increased ionising dose/neutron fluence ratio utilised in the MTR experiments, compared with AGR conditions, may have caused a more progressive 'breakthrough' into the CPV contained within the filler particles. This could reveal larger total intruded volume for the exvirgin oxidised samples when compared to ex-AGR specimens at similar weight loss. This would concur with the increased intrusion seen in the low pressure regions of the curve, but it is impossible to infer whether the observed increase, visible in Figure 5, is due to additional porosity created from either the binder or filler phases. Deconvoluting these phenomena would be highly complex, as the high pressure intrusion, which is thought to be partially contributed to by the presence of CPV, could overshadow this effect. 
Ex-virgin oxidised samples trend with increasing weight loss, where the highest mass loss sample showed the largest volume contributions across the range of pore-throat entrance sizes. All samples, apart from M870, showed secondary intrusion at the highest operating pressures (SI Figure 4). Additionally, in sample M870, the change in slope instead appeared abruptly at a much lower operating pressure, and contributed a sharp volume increase, compared to the continuous intrusion observed for the other samples. This could allude to a secondary mechanism deforming the internal network, triggered by a sudden rearrangement of the internal microstructure, in order to accommodate the additional volume. This is supported when looking at the extrusion curves in Figure 4 shown in the Supplementary Information. Large hysteresis was observed at high pressures in the low weight loss samples which corresponds to trapped or 'snapped' mercury within the complex void system. However, in sample M870, elastic compression of the sample was instead observed at the highest pressures. This suggested that with increased porosity, the compressibility of the material was compromised.

\subsubsection{Ex-AGR specimens}

Looking more closely at the Ex-AGR samples in Figure 6 revealed that the low weight loss specimens $(<15 \%)$ asymptote towards similar total intruded volumes accessed by mercury, with the exception on M671. These percolation curves show intrusion that is highly comparable to that observed for the virgin specimen, 921-2A. The measured cumulative fast neutron dose for each of these samples were similar ( $\pm 4 \mathrm{dpa}$ ) and the weight loss only varied by $\sim 4 \%$ and therefore, it is reasonable to expect these samples to have similar percolation curves and supports the reliability of the methodology.

Sample 0M55 (6) displays comparable intrusion to the ex-virgin, oxidised sample M870 (5), where at a pressure of $0.3 \mathrm{MPa}$ the curve shows abrupt, vertical intrusion equating to an increased volume of $0.015 \mathrm{~cm}^{3} \mathrm{~g}^{-1}$. Both samples show comparable weight loss, within $1.8 \%$, whilst the cumulative fast neutron dose was significantly higher (due the accumulation of fast neutron dose in both AGR and MTR conditions) for sample 0M55. It was therefore proposed that at weight loss values around $\sim 25 \%$ the internal microstructure of Gilsocarbon was compromised, and the network of voids become susceptible to 
sudden breakage, and less accommodating to the continuous intrusion observed in virgin and low weight loss samples.

\section{Conclusions}

Despite the complexity and heterogeneity of Gilsocarbon graphite at all length scales, and the difficulty of studying irradiated samples, experimental trends have been derived that give insights into the mechanism and effects of radiolytic oxidation. Characterisation of the samples with a bespoke helium micropycnometer has allowed quantification of gas-accessible and isolated pore volumes, and the subsequent changes that occur to these values with increasing weight loss and cumulative fast neutron dose. Results of the experimental measurements showed a general increase of skeletal density across the weight loss series. This suggests a decrease in CPV, assumed to be caused by a combination of factors, such as the opening of CPV by oxidation, as well as the closure of CPV driven by the crystal dimensional change upon irradiation. This was further supported by the calculated values of OPV and CPV. In virgin specimens there were similar volumes of OPV and CPV. Radiolytic oxidation caused the progressive evolution of the OPV, which increased linearly with weight loss. The decrease in $\mathrm{CPV}$ upon radiolytic oxidation gives a smaller contribution to the overall void volume change.

A uniform evolution of the pore space, with respect to pore-throat diameter, was apparent when comparing mercury intrusion characteristics. Specifically, the open pore volume developed over the pore-throat diameter range of $\sim 2$ $100 \mu \mathrm{m}$. This is in accord with previous image analysis of microscopy images that showed that radiolytic gasification produces a large increase in the number of pores around $100 \mu \mathrm{m}$.

The results of the present study give support to recent postulates that radiolytic oxidation is a surface driven phenomena which attacks the void space uniformly. They contradict historical 'Reactive Pore Volume' (RPV) models utilised in the nuclear industry which predicted that pores larger than $2 \mu \mathrm{m}$ diameter would become ineffective in the oxidation process due to a higher probability of deactivation, by interacting with another excited species, before reaching the pore wall. 
The deductions within this work are semi-quantitative. In further work, computational modelling will be used to generate quantitative void structure models at every oxidation stage. Those will be used to make a definitive judgement on the RPV model, and to provide data for inputting to current larger scale models of reactor core behaviour such as FEAT-DIFFUSE. 


\section{Acknowledgements}

The financial support and advice of EDF Energy (Barnwood, Gloucester, UK) is gratefully acknowledged. However, the views expressed in this paper are those of the authors and do not necessarily represent the views of the sponsors.

\section{Appendix A. Supplementary materials}

Supplementary data associated with this article can be found in the on-line version at ...

\section{References}

[1] D. Norfolk, Gas chemistry in nuclear reactors and large industrial plant, Nucl. Energy 19 (4) (1980) 253-256.

[2] J. Best, W. Stephen, A. Wickham, Radiolytic graphite oxidation, Prog Nucl Energ 16 (2) (1985) 127-178.

[3] V. Labaton, B. Ashton, R. Lind, J. Tait, The effect of variation in pore size on the rate of radiolytic corrosion of graphite by carbon dioxide, Carbon 7 (1) (1969) 59-75.

[4] N. Murdie, I. A. Edwards, H. Marsh, Changes in porosity of graphite caused by radiolytic gasification by carbon dioxide, Carbon 24 (3) (1986) 267-275.

[5] Z. Mileeva, D. Ross, S. King, A study of the porosity of nuclear graphite using small-angle neutron scattering, Carbon 64 (2013) 20-26.

[6] E. D. Eason, G. N. Hall, B. J. Marsden, G. B. Heys, Models of bending strength for Gilsocarbon graphites irradiated in inert and oxidising environments, J. Nucl. Mater. 436 (1) (2013) 208-216.

[7] Y. Vertyagina, T. J. Marrow, Multifractal-based assessment of Gilsocarbon graphite microstructures, Carbon 109 (2016) 711-718.

[8] J. Best, C. Wood, The radiolytic graphite/CO2 reaction. The effect of pore structure on corrosion in gas mixtures containing carbon monoxide, methane and water, Carbon 13 (6) (1975) 481-488. 
[9] J. Hutcheon, H. Cowen, N. Godwin, The Role of Pore Structure in the Oxidation of Graphite by Irradiated Carbon Dioxide, UK Atomic Energy Authority, 1962.

[10] R. Krishna, A. N. Jones, B. J. Marsden, Transmission electron microscopy, Raman and X-ray photoelectron spectroscopy studies on neutron irradiated polycrystalline graphite, Radiation Physics and Chemistry 107 (2015) 121127 .

[11] P. C. Minshall, I. A. Sadler, A. J. Wickham, Radiolytic graphite oxidation revisited (IAEA-TECDOC-901.) (1996) 181.

[12] Z. Zhou, W. Bouwman, H. Schut, T. van Staveren, M. Heijna, C. Pappas, Influence of neutron irradiation on the microstructure of nuclear graphite. An X-ray diffraction study, J. Nucl. Mater. 487 (2017) 323-330.

[13] G. Hall, B. Marsden, S. Fok, The microstructural modelling of nuclear grade graphite, J. Nucl. Mater. 353 (1) (2006) 12-18.

[14] W. H. Huang, S. C. Tsai, C. W. Yang, J. J. Kai, The relationship between microstructure and oxidation effects of selected IG- and NBG-grade nuclear graphites, J. Nucl. Mater. 454 (1) (2014) 149-158.

[15] B. Šavija, G. E. Smith, P. J. Heard, E. Sarakinou, J. E. Darnbrough, K. R. Hallam, E. Schlangen, P. E. Flewitt, Modelling deformation and fracture of Gilsocarbon graphite subject to service environments, J. Nucl. Mater 499 (2018) 18-28.

[16] T. Trevethan, M. Heggie, Molecular dynamics simulations of irradiation defects in graphite. Single crystal mechanical and thermal properties, Comput. Mater. Sci. 113 (2016) 60-65.

[17] R. Telling, M. Heggie, Radiation defects in graphite, Philosophical Magazine 87 (31) (2007) 4797-4846.

[18] B. März, K. Jolley, T. J. Marrow, Z. Zhou, M. Heggie, R. Smith, H. Wu, Mesoscopic structure features in synthetic graphite, Materials and Design 142 (2018) 268-278. 
[19] M. Fahad, K. McNally, T. Yates, N. Warren, B. Marsden, P. Mummery, G. Hall, Finite element modelling of multilayer Advanced Gas-cooled Reactor bricks and creep interaction, Nuclear Engineering and Design 324 (2017) 390-401.

[20] B. J. Marsden, G. N. Hall, O. Wouters, J. Vreeling, J. Van der Laan, Dimensional and material property changes to irradiated Gilsocarbon graphite irradiated between 650 and $750{ }^{\circ} \mathrm{C}$, vol. 381, 62-67, the Seventh and Eighth International Graphite Specialists Meetings (INGSM), 2008.

[21] H. Freeman, A. Jones, M. Ward, F. Hage, N. Tzelepi, Q. Ramasse, A. Scott, R. Brydson, On the nature of cracks and voids in nuclear graphite, Carbon 103 (2016) 45-55.

[22] Y. Vertyagina, T. J. Marrow, 3D Cellular Automata fracture model for porous graphite microstructures, Nuclear Engineering and Design 323 (2017) 202-208.

[23] G. M. Laudone, C. M. Gribble, G. P. Matthews, Characterisation of the porous structure of Gilsocarbon graphite using pycnometry, cyclic porosimetry and void-network modeling, Carbon 73 (2014) 61-70.

[24] G. M. Laudone, C. M. Gribble, G. P. Matthews, K. L. Jones, Measurement and modelling of void structure and diffusion processes within Gilsocarbon Graphite, in: P. Flewitt, A. Wickham (Eds.), The 4th EDF Energy Nuclear Graphite Symposium. Engineering Challenges Associated with the Life of Graphite Reactor Cores, EMAS Publishing, 2015.

[25] J. M. Dickinson, J. W. Shore, Observations concerning the determination of porosities in graphites, Carbon 6 (6) (1968) 937-941.

[26] K. L. Jones, G. M. Laudone, G. P. Matthews, A multi-technique experimental and modelling study of the porous structure of IG-110 and IG-430 nuclear graphite, Carbon 128 (2018) 1-11.

[27] D. J. Baker, J. B. Morris, Structural damage in graphite occuring during pore size measurements by high pressure mercury, Carbon 9 (5) (1971) $687-690$. 
[28] F. Rodríguez-Reinoso, J. Rouquerol, K. Unger, K. S. Sing, Characterization of Porous Solids III, vol. 87, Elsevier, 1994.

[29] G. P. Matthews, C. L. Levy, G. M. Laudone, K. L. Jones, C. J. Ridgway, I. L. Hallin, S. A. Gazze, L. Francis, W. R. Whalley, J. Schoelkopf, P. A. C. Gane, Improved interpretation of mercury intrusion and soil water retention percolation characteristics by inverse modelling and void cluster analysis, Transp Porous Med 124 (2018) 631-653.

[30] B. Marsden, A. Jones, G. Hall, M. Treifi, P. Mummery, 14 - Graphite as a core material for Generation IV nuclear reactors, in: P. Yvon (Ed.), Structural Materials for Generation IV Nuclear Reactors, Woodhead Publishing, 495-532, 2017.

[31] K. Sing, Characterization of porous materials past present and future, Colloids and Surfaces A 241 (1) (2004) 3-7, ISSN 0927-7757.

[32] J. van Brakel, S. Modry, M. Svata, Mercury Porosimetry. State of the Art, Powder Technol. 29 (1981) 1-12.

[33] B. Kelly, P. Johnson, P. Schofield, J. Brocklehurst, M. Birch, U.K.A.E.A. northern division studies of the radiolytic oxidation of graphite in carbon dioxide, Carbon 21 (4) (1983) 441-449.

[34] S. D. Preston, W. E. Ellis, Techniques for Measuring the Properties of Unirradiated and Irradiated AGR Graphite, in: G. B. Neighbour (Ed.), Management of Ageing in Graphite Reactor Cores, 2007.

[35] P. J. Hacker, A study of the coefficient of thermal expansion of nuclear graphites, Ph.D. thesis, University of Bath, 2001.

[36] A. Wickham, Revisiting AGR graphite weight-loss predictions: a first principles review of the basis and application of the FEAT-DIFFUSE code, in: The 5th EDF Energy Nuclear Graphite Symposium: Science and Engineering in Collaboration to Support Safe Operation of the Graphite Reactor Cores. Emas Publishing, Warrington, 2016.

[37] M. R. Bradford, A. G. Steer, A structurally-based model of irradiated graphite properties, J. Nuc. Mat. 381 (1) (2008) 137 - 144. 
[38] K. Shen, S. Yu, F. Kang, The microstructure and texture of Gilsocarbon graphite, Carbon 153 (2019) 428 - 437.

[39] P. Heard, M. Wootton, R. Moskovic, P. Flewitt, Deformation and fracture of irradiated polygranular pile grade A reactor core graphite, J. Nucl. Mater. 418 (1) (2011) 223-232.

[40] D. Liu, B. Gludovatz, H. S. Barnard, M. Kuball, R. O. Ritchie, Damage tolerance of nuclear graphite at elevated temperatures, Nat. Commun. 8 (15942). 